\title{
Noise Measurement by Echo Integrator
}

\author{
Yoshimi Takao and Masahiko Furusawa \\ National Research Institute of Fisheries Engineering, Ebidai, Hasaki, Kashima, \\ Ibaraki 314-04, Japan
}

(Received December 14, 1994)

\begin{abstract}
A method to measure noise at the output of echo integrators has been developed for precise estimation of fisheries resources by acoustic means. This method is more practical than the method to measure noise at receiver output, because noise contribution can be easily compared with the volume backscattering strength (SV) of fish and because there is less ambiguity in noise measurement. Also a conversion equation of the echo integrator output to the equivalent noise spectrum level is derived. This facilitates the comparison, for example, between different echo sounding system. We applied this method to a dual beam echo sounding system on board research vessel Kaiyo-maru, and confirmed that the difference in noise SV of $5 \mathrm{~dB}$ agreed with the theoretical prediction.
\end{abstract}

Key words: acoustic survey, noise, echo integration, noise spectrum level

Noise is an important problem for hydroacoustic surveys of fisheries resources, because it may cause a large error in estimated results and shorten the detectable range of fish.

Noise can be classified into low frequency noise (audible by fish) and high frequency noise (having an effect on echo sounders). ${ }^{1}$ Low frequency noise generated by a survey vessel generally causes fish to avoid the vessel, resulting in an underestimated fish abundance. High frequency noise usually causes acoustic variables, such as volume backscattering strength (SV), to be overestimated, but sometimes a noise threshold level that is too high can cause an underestimation.

To ensure that hydroacoustic surveys are precise and accurate, it is necessary to estimate and remove the effects of noise. We examined two different methods of measuring high frequency noise. A simple method is observing noise at the receiver output, but this method has difficulty with defining average noise level due to variable characteristics of the noise. Therefore, we introduced a more practical method whereby noise is measured at the echo integrator output.

At first, we examined the above methods theoretically. Next, we measured the noise level by the echo integrator on board a research vessels and confirm effectiveness of this method.

\section{Theory}

\section{Direct Measurement of Noise Level}

The noise pressure amplitude $P_{N}$ at the transducer surface is shown by

$$
P_{N}=P_{S} D_{S}(1 / R) D
$$

where $P_{S}$ is pressure at a reference range from a noise source, $D_{S}$ is directivity of the noise source, $R$ is range between a transducer and the noise source, and $D$ is the directivity of receiving transducer of an echo sounder.

To derive the received noise spectrum level, we measure the echo amplitude $E_{R}$ at the preamplifier output shown by

$$
E_{R}=P_{N} M G_{R}
$$

where $M$ is receiving sensitivity of the transducer and $G_{R}$ is preamplifier gain. From Eq. (2), the received spectrum level of noise is

$$
P_{N} / \Delta f^{1 / 2}=E_{R} /\left(M G_{R} \Delta f^{1 / 2}\right)
$$

where $\Delta f$ is bandwidth of the receiver.

Equation (3) shows that we can estimate the noise spectrum level by measuring $E_{R}$. But it is difficult to measure $E_{R}$ directly under a clear definition (and therefore to estimate the noise level), because the noise level generally varies drastically. It depends on vessel motions, etc. and sometimes changes in one ping duration.

\section{Noise Measurement by Echo Integrator}

The output of $20 \log r$ TVG-amplifier of a quantitative echo sounder, $E_{T}$, is shown by

$$
E_{T}=E_{R} G_{T} r \exp (2 \alpha r)
$$

where $r$ is range, $G_{T}$ is the coefficient for $20 \log r$ TVG-amplifier gain, and $\alpha$ is an absorption attenuation coefficient.

The echo integrator output for a layer between $r$ and $r+r_{w}$ is shown by

$$
\left\langle S_{V}\right\rangle=\frac{1}{K^{2}} \frac{1}{r_{w}} \int_{r}^{r+r_{w}}\left\langle E_{T}^{2}\right\rangle \mathrm{d} r
$$

where $\left\langle S_{V}\right\rangle$ is average volume backscattering strength, $\left\langle E_{T}{ }^{2}\right\rangle$ is ensemble average of $E_{T}{ }^{2}, r_{w}$ is the width of an integration layer, and $K$ is the multiple echo coefficient of an echo sounder system defined by

$$
K^{2}=\left(P_{0} M G_{R} G_{T}\right)^{2}(c \tau / 2) \Psi
$$

where $P_{0}$ is source pressure, $c$ is sound speed, $\tau$ is effective pulse width and $\Psi$ is equivalent beam angle.

The TVG output of noise is from Eqs. (2) and (4)

$$
E_{T}=P_{N} M G_{R} G_{T} r \exp (2 \alpha r) .
$$

From Eqs. (5), (6), and (7), the echo integrator output of the noise ("noise SV") is 


$$
\left\langle S_{V}\right\rangle_{N}=\frac{P_{N}^{2}}{P_{0}^{2}} \frac{2}{c \tau} \frac{1}{\Psi} r^{2} \exp (4 \alpha r) g
$$

where

$$
\begin{aligned}
g & =\left[\int_{r}^{r+r_{w}} r^{2} \exp (4 \alpha r) \mathrm{d} r / r_{w}\right] /\left[r^{2} \exp (4 \alpha r)\right] \\
& =1 /\left(32 \alpha^{3} r_{w} r^{2}\right)\left[8 \alpha^{2}\left(r_{2}^{2} h-r^{2}\right)-4 \alpha\left(r_{2} h-r\right)+h-1\right] \\
h & =\exp \left(4 \alpha r_{w}\right) \\
r_{2} & =r+r_{w} .
\end{aligned}
$$

The coefficient $g$ is the deviation from the ordinary range characteristics of $20 \log r$ TVG, introduced by integration process. As can be seen from the first expression of Eq. (9), when $r$ is large and $r_{w}$ is small, $g$ is nearly unity; for example $g=1.16$ for $r=200 \mathrm{~m}, r_{w}=20 \mathrm{~m}$, and $\alpha=10 \mathrm{~dB} / \mathrm{km}$.

If we assume that (1) the bandwidth of the receiver is narrow and noise spectrum is flat in the band, and (2) the noise is received omni-directionally, then the relationship between noise pressure and noise spectrum level $\left(N_{P}\right)$ is

$$
P_{N}^{2}=N_{P}^{2} \frac{\Delta f}{D_{I}}
$$

where $D_{I}$ is the directivity index of a receiving transducer.

Equivalent noise spectrum level is obtained by using Eqs. (8) and (10) as follows:

$$
N_{P}^{2}=\frac{P_{0}^{2} D_{I} c \tau \Psi}{2 \Delta f r^{2} \exp (4 \alpha r) g}\left\langle S_{V}\right\rangle_{N} .
$$

This is the conversion equation of the output of echo integrator to the equivalent noise spectrum level.

Although we assumed in Eq. (10) that the noise was omni-directional (for example noise from the screw propeller would invalidate this assumption), this equivalent $N_{P}$ can be used effectively, because we can compare it easily with other kinds of noises. ${ }^{2)}$ Also, by measuring noise with an echo integrator, we can easily measure average noise power and compare results with fish backscattering strength.

\section{Measurements}

\section{Acoustic System}

We conducted noise measurements using our quantitative echo sounding system which has the dual-beam echo integration function. ${ }^{3,4)}$ Table 1 lists its specifications and Fig. 1 shows the system block diagram.

The transducer is mounted on a V-fin type towed body to reduce error caused by noise, bubbles, and transducer motion. The receiver has four independent TVG channels, that is for narrow and wide beams and for $20 \log r$ and 40 $\log r$ TVG characteristics. The outputs of $20 \log r$ TVG are transferred to the echo integrator and the outputs of 40 $\log r$ TVG are transferred to the dual beam processor. The echo integrator has narrow and wide channels to perform

\begin{tabular}{|c|c|c|c|}
\hline NARROW & COMMON & WIDE & \\
\hline Frequency & & $38 \mathrm{kHz}$ & \\
\hline Transducer & & 79 PZT elements & \\
\hline $\begin{array}{l}\text { Effective } \\
\text { transducer radius }\end{array}$ & $19 \mathrm{~cm}$ & & $7 \mathrm{~cm}$ \\
\hline Beam width & $6.4^{\circ}$ & & $15.6^{\circ}$ \\
\hline $\begin{array}{l}\text { Equivalent } \\
\text { beam angle }\end{array}$ & $0.0072 \mathrm{sr}$ & & $0.0120 \mathrm{sr}^{*}$ \\
\hline Transmitting power & & $2.8 \mathrm{~kW}$ & \\
\hline Pulse duration & & $0.6,1.2,2.4 \mathrm{~ms}$ & \\
\hline Depth range & & $50,100,200,400,800 \mathrm{~m}$ & \\
\hline TVG & & $\begin{array}{c}20 \log r+2 \alpha r \\
40 \log r+2 \alpha r\end{array}$ & \\
\hline$\alpha$ & & $10.0 \mathrm{~dB} / \mathrm{km}$ & \\
\hline
\end{tabular}
independent integration. ${ }^{4)}$

\section{Method of Noise Integration}

We measured noise levels received by our system several times during the survey to examine characteristics of noise and to get information on setting threshold parameters of
Table 1. Specifications of the echo sounding system

* Composite beam.

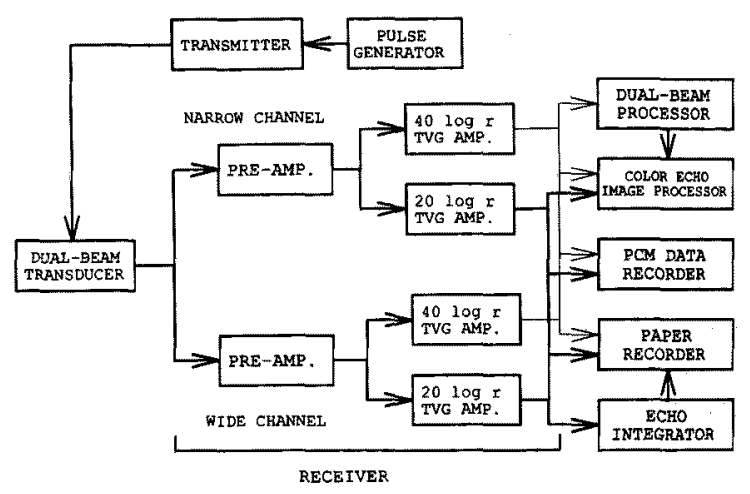

Fig. 1. System block diagram of echo sounding system.

the echo integrator. The effect of engine speed or ship speed upon noise was measured before and after each survey.

To measure noise, we stopped transmitting and only receive noise. Because TVG outputs of the noise are low at small ranges and increase with the range (see Eq. (7)), we started noise integration at depths greater than $200 \mathrm{~m}$ where noise contributions are well above our system's minimum detectable SV of $-99.9 \mathrm{~dB}$. We did not use the threshold function.

\section{Example of Noise Measurement}

Figure 2 shows results of noise measurements conducted in 1988 off Tateyama, Chiba prefecture, by an echo integrator aboard the research vessel former "Kaiyo maru" $(\mathrm{L} \times \mathrm{B} \times \mathrm{D}=84.7 \times 15.0 \times 9.2 \mathrm{~m}, 3210 \mathrm{t})$. The $\mathrm{Y}$-axis shows noise SV in a layer between 250 and $300 \mathrm{~m}$, and the X-axis shows ship speed. The echo integration period was one minute. Black circles are noise SV obtained from the narrow channel data (narrow SV) and the white squares are wide SV. Solid and broken regression lines were derived for the narrow and wide SV, respectively, using a least squares method. Though the variance of the integrator outputs was large because of a short integration period, we observed a trend whereby increasing ship speed resulted in increased noise. Figure 3 shows an equivalent noise spectrum level converted from noise SV using Eq. (11). Noise spectrum level rose with increased ship speed. 


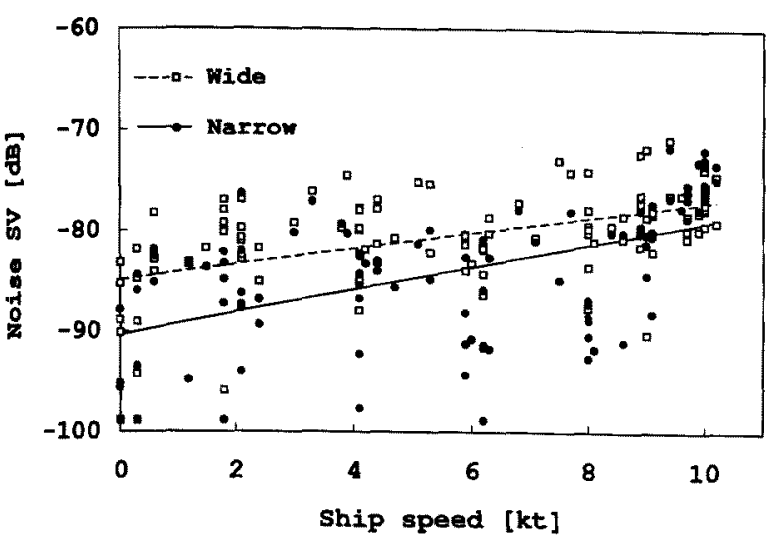

Fig. 2. The results of "noise integration" on board R/V "Kaiyo maru" on 15 November 1988 off Tateyama.

The depth layer for noise SV is between 250 and $300 \mathrm{~m}$. White squares are for wide channel SV and black circles are for narrow channel SV. The solid and broken lines are regression lines for the narrow and wide SV data, respectively.

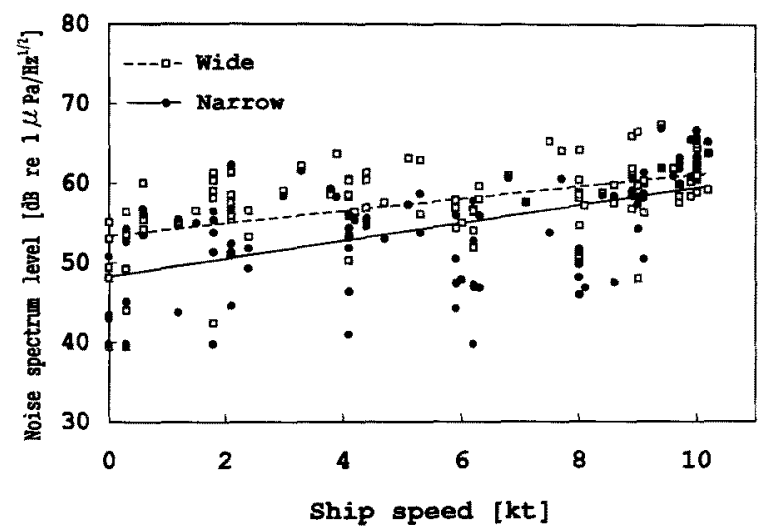

Fig. 3. Equivalent noise spectrum level converted from noise SV of Fig. 2 as a function of ship speed.

\section{Discussion}

We showed an equation that estimates noise spectrum level by directly measuring $E_{R}$ at the receiver output (Eq. (3)). This method, however, has difficulty with defining average noise level due to variable characteristics of the noise. Measuring noise with an echo integrator is easier and more practical than measuring noise directly at the receiver output, because one can measure the average power of the noise and can easily compare the contributions of noise with the backscattering strength of fish.

During the acoustic surveys we often conducted noise integration for a short time, then compared the noise SV with fish SV. When the noise SV was relatively high, we changed noise threshold parameters and conduct noise integration again. In this way, we could examine the effect of threshold parameters. If a threshold level that is too high was required, we slowed down the ship.

As an application of this method, we incorporated the equivalent noise spectrum level as a design specification of new research vessels. $\left.{ }^{5}\right)$

Here we discuss the difference of the noise SV in narrow and wide channels. From Eqs. (8) and (10), the ratio of the noise SV of narrow to wide channels is ${ }^{4)}$

$$
\frac{\left\langle S_{V}\right\rangle_{N N}}{\left\langle S_{V}\right\rangle_{N W}}=\frac{\Delta f_{N}}{\Delta f_{W}} \frac{D_{I W}}{D_{I N}} \frac{\Psi_{C}}{\Psi_{N}}
$$

where $\Psi_{C}$ is the equivalent beam angle for the composite narrow and wide beam, and subscripts $N$ and $W$ represent narrow and wide channels, respectively. We substitute the approximate directivity index and the equivalent beam angle of a circular piston transducer ${ }^{6}$ into Eq. (12). The band width of our receivers are $3 \mathrm{kHz}$ and are equal for narrow and wide channels. The result shows that the noise SV of the wide channel is theoretically about $5 \mathrm{~dB}$ higher than the narrow one for our echo sounding system. This value agrees with the difference of the regression curves when the "Kaiyo maru" was drifting or sailing at low speed (see Fig. 2).

The noise SV of both narrow and wide channels increases as ship speed increased (see Fig. 2). This is because omni-directional ambient noise is superior at low ship speed and propeller noise increases as ship speed increases. Figure 2 also shows that the narrow noise SV increased more rapidly with ship speed and varied more significantly than did the wide SV. An explanation for these differences may be that the propeller noise has radiating directivity and its pattern and noise spectrum level change with speed, ${ }^{1)}$ and the weight of the propeller noise in total noise is larger for the narrow channel than the wide channel.

Towing speed affected our towed body position. We conducted an experiment to examine the relationship between noise SV and towed body positions. Although analysis of these data has not yet completed, it should help us to explain this relationship.

\section{Conclusion}

We introduced a method to measure noise using an echo integrator. We examined this method theoretically and experimentally.

This method is more practical than the directly measuring noise at the receiver output because one can measure the average power of noise and can easily compare the contributions of noise with the backscattering strength of fish.

We derived a conversion equation of the echo integrator output to the equivalent noise spectrum level which facilitates comparison among several kinds of noises and echo sounder systems.

As part of our acoustic surveys, we often conduct noise integration for a short time, then determine suitable threshold parameters.

Acknowledgments We thank Kouichi Sawada and Youichi Miyanohana, National Research Institute of Fisheries Engineering, for their support to this study. We are grateful to Neal Williamson and Steve de Blois, Alaska Fisheries Science Center, NOAA, USA, for reviewing and refining the manuscript.

\section{References}

1) R. B. Mitson: Ship noise related to fisheries research. Proc. Inst. of Acoust., 11 (Part 3), 61-67 (1989).

2) G. M. Wenz: Acoustic ambient noise in the ocean. $J$. acoust. Soc. Am., 34, 1936-1956 (1962)

3) M. Furusawa, Y. Takao, K. Sawada, T. Oukubo, and K. Yamatani: 
Versatile Echo Sounding System Using Dual Beam. Nippon Suisan Gakkaishi, 59, 967-980 (1993).

4) Y. Takao and M. Furusawa: Dual-beam echo-integration method for precise estimation of fish biomass. Proc. 4th Pacific Con. Mar. Sci. Tech. (PACON), Vol. I, 499-506 (1990).
5) M. Furusawa and Y. Takao: Acoustic Specifications of Fisheries Research Vessel 'Kaiyo-maru', the Joint Session of FTFB and FAST Working Groups, Bergen, Norway, 1992, pp. 1-17.

6) C. S. Clay and H. Medwin: Acoustical Oceanography, Wiley, New York, 1977, pp. 147-148. 